\title{
Improvement of Adult BTE Hearing Aid Wearers' Front/Back Localization Performance Using Digital Pinna-Cue Preserving Technologies: An Evidence-Based Review
}

\author{
Jingjing $\mathrm{Xu}^{1}$ and Woojae $\mathrm{Han}^{2}$ \\ ${ }^{1}$ School of Communication Sciences and Disorders, University of Memphis, Memphis, TN, USA \\ ${ }^{2}$ Division of Speech Pathology and Audiology, Research Institute of Audiology and Speech Pathology, College of Natural Sciences, \\ Hallym University, Chuncheon, Korea
}

\section{Received September 6, 2014 \\ Revised October 30, 2014 \\ Accepted October 30, 2014}

\section{Address for correspondence Jingjing $\mathrm{Xu}, \mathrm{PhD}$ \\ School of Communication Sciences and Disorders, University of Memphis, 807 Jefferson Ave, Memphis, TN 38105 , USA \\ Tel $+1-901-678-5800$ \\ Fax $+1-901-525-1282$ \\ E-mail jxu3@memphis.edu}

This systematic review evaluated the impact of using digital pinna-cue preserving technologies (PPT) on front/back sound localization for adult hearing aid users. Two peer-reviewed studies and two non-peer-reviewed studies were included. Lab-based and self-report outcomes were both assessed. The overall findings suggested that PPT was superior to omni-directional and full directional settings in a relatively quiet, well-controlled laboratory environment but not in the real world. However, observed individual differences in self-report measures suggested that PPT was potentially beneficial to certain hearing aid users. PPT candidacy was discussed and the importance of a pre-fitting interview/consultation was emphasized to assist clinicians in making a solid evidence-based and cost-effectiveness decision when prescribing hearing aids to adults with hearing impairment.

Korean J Audiol 2014;18(3):97-104

KEY WORDS: Pinna-cue · High frequency directivity · Hearing aids · Localization · Systematic review.

\section{Introduction}

Sound localization is an important function of human hearing. It allows listeners to accurately localize the direction of incoming sounds in their everyday listening and communication. With this ability, listeners can construct auditory images and have a sense of the environment they are in. It is especially critical for a listener to know the direction of surrounding sounds in certain situations for safety reasons. For instance, when walking across a street, it is important to be able to hear from which direction motor vehicles are coming. In addition, localization is an important hearing aid outcome, which is closely relates to hearing aid satisfaction. ${ }^{1)}$ Therefore, sound localization has received considerable research attention from both clinicians and hearing scientists.

How do human beings localize sounds? Three types of acous-

This is an Open Access article distributed under the terms of the Creative Commons Attribution Non-Commercial License (http://creativecommons. org/licenses/by-nc/3.0/) which permits unrestricted non-commercial use, distribution, and reproduction in any medium, provided the original work is properly cited. tic cues contribute to this ability. They are interaural level differences (ILDs), interaural time differences (ITDs), and monaural spectral cues. Details of these three cues can be found in Blauert. ${ }^{2)}$ Briefly, ILDs are based on the loudness difference between the right and the left ears and they are dominant for high frequency sounds (above $1500 \mathrm{~Hz}$ ) due to the head shadow effect. ITDs are based on the difference in arrival time between the two ears and they are dominant for low frequency sounds below $800 \mathrm{~Hz}$. Monaural spectral cues are used to localize the front/back location of a sound source on a horizontal plane and the elevation of a sound source. A pinna (or auricle) provides monaural spectral cues, together with the head and the torso of the listener, to resolve front/back confusions on the horizontal plane and for elevation hearing. In this review, we focus on localization cues that relate to the use of a pinna.

The ability to accurately localize incoming sounds for an adult listener can be degraded by various factors. Hearing loss is one of the major factors, which has a detrimental impact on older adults. When audibility is compromised due to hearing impairment, localization cues are distorted, resulting in poorer 
localization performance compared to listeners with normal hearing. Moreover, ILD cues can be severely deteriorated by asymmetrical hearing loss. Further, listeners with high frequency hearing loss may not be able to utilize the spectral cues for front/back and elevation localization because they receive insufficient high frequency spectral cues.

Hearing aids are commonly used by listeners with hearing loss to restore audibility of residual hearing. However, existing research has shown that aided localization performance is poorer than unaided localization performance. ${ }^{3,4)}$ The acoustic cues for localizing sounds are altered by certain factors related to hearing aid use, such as amplification, ${ }^{3)}$ physical design of the hearing aids, ${ }^{5,6)}$ and actual hearing aid features. ${ }^{7)}$ As mentioned previously, a pinna provides substantial amount of monaural spectral cues. Placement of the hearing aid microphone can have a large impact on the availability of these cues. Acoustic cues delivered by a pinna are largely maintained when inthe-ear, in-the-canal, or completely-in-the-canal hearing aids are used because the microphone of these hearing aids is located at the concha or in the ear canal, where incoming sounds are picked up after they are filtered by the pinna. In contrast, when using a behind-the-ear (BTE) style hearing aid, incoming sounds are picked up by the hearing aid microphones before the sounds reach the pinna. Therefore, monaural spectral cues are compromised, which may then result in poor front/ back and up/down localization performance. Previous research has shown that better aided front/back localization performance occurs with microphones that are placed in the concha or ear canal compared to microphones placed above the ear. ${ }^{5)}$ Sivonen $^{8)}$ measured the binaural directivity patterns for these two microphone placements on an artificial head. Sizeable directivity advantages were observed at frequencies above 1000 $\mathrm{Hz}$ when the microphone was located at the entrance to the ear canal. This finding indicated the importance of preserving the acoustic cues afforded by the pinna for the most accurate aided localization performance.

Despite the fact that using BTE hearing aids may result in poor front/back and up/down localization performance, BTE users have been increasing rapidly in recent years because of their advantages of having more space for housing powerful electronic components and using larger batteries. ${ }^{9)}$ Moreover, in addition to traditional BTE hearing aids with standard earhooks and earmolds, open-fit BTE hearing aids and receiverin-the-canal BTE hearing aids using slim tubing have become popular. A report in 2009 showed that $63.4 \%$ of hearing aids sold in the United States were BTEs. ${ }^{10)}$ This number has been increased to $69.9 \%$ in $2011 .{ }^{11)}$ Because the microphone placement effect of BTE hearing aids distorts the monaural spectral cues coming from the pinna, researchers and engineers are attempting to restore the effect of pinna using digital signal processing. They hope that the compensation of monaural spectral cues will improve front/back and up/down localization.

Currently, a common way to digitally restore the effect of pinna to BTE hearing aids is to manipulate the polar patterns of the multi-channel directional microphone. Basically, the polar patterns for low frequency channels are set to an omni-directional mode, while the polar patterns for high frequency channels (usually above $1000 \mathrm{~Hz}$ ) are set to a front-facing directional mode (e.g., hypercardioid). Thus, this feature also is called high frequency directionality. By introducing such directional microphone settings, the directivity index is similar to that of an unoccluded ear (Fig. 1). Several hearing aid manufacturers have employed this feature in many of their hearing aids now on the market. These manufacturers market this technology differently. Examples are ReSound split-band directionality, Phonak Real Ear Sound, Siemens TruEar, and Widex Digital Pinna. Clinicians may run into a question of whether this technology is effective and whether they should prescribe hearing aids with this feature to their patients. Given the fact that this technology has been around for nearly 10 years, evidence of whether it is effective is surprisingly sparse. There is a need to perform an evidence-based study to provide audiologists with further evidence for prescribing this feature. The research question to be explored in this paper is: does the existing evidence support prescribing hearing aids with pinna-cue preserving technologies (PPT) to improve front/back discrimination on a horizontal plane? Henceforth, PPT will refer herein to the pinna-cue preserving technologies used in different BTE hearing aids.

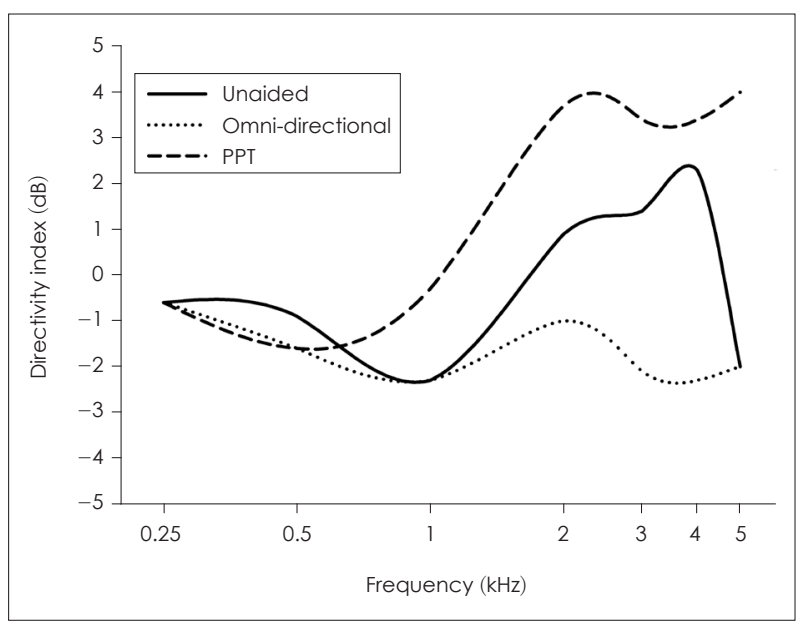

Fig. 1. A demonstration of Directivity Index for (1) unoccluded ear, (2) Omni-directional microphone, and (3) PPT (cutoff frequency of $1 \mathrm{kHz}$ ) measured with a KEMAR in an anechoic chamber. Adapted from Keidser, et al. Int J Audiol 2009;48:789-803. ${ }^{17)}$ PPT: pinna-cue preserving technologies. 


\section{Literature Review}

Different technical terms have been used to denote PPT, such as split-band directionality, high frequency directionality, and frequency dependent microphone. Thus, a variety of key words were used for literature search, including "frequency dependent microphone," "high-frequency directionality," "split-band directionality," "pinna," "hearing aids," and "localization." Since the published research on this topic is already known to be limited, a broad literature review was conducted using the key words: "pinna," "hearing aids," and "localization" to gather as many related research studies as possible. This search in Pubmed found 24 articles, but only one was retained after a full abstract review. From the reference list of this article, one more article was identified. With the same three key words, a separate search in CINAHL was performed. Four more articles were identified. However, none of these were retained after reviewing their abstracts.

Since only two peer-reviewed articles were identified, the literature search was expanded to non-peer-reviewed articles. Despite the fact that they are not reviewed by experts, useful information could still be extracted from non-peer-reviewed articles as evidence as long as they have good control of their experimental designs. Rationales for the inclusion of non-peer reviewed articles were described in articles by $\operatorname{Cox}^{12)}$ and Keidser. ${ }^{13)}$ Another run of search in Google Scholar was conducted using the key words "frequency dependent microphone," "highfrequency directionality," and "split-band directionality." Four non-peer-reviewed journal articles were identified. After reviewing their abstracts, two were excluded because one focused on sound quality when using PPT and the other focused on the benefits of using PPT relative to that of unaided hearing.

After the literature search, a total of four articles were included in this evidence-based review and they are summarized in Table 1. Outcome measures for this review included laboratory-based localization measurements and self-report measures. The following section evaluates each retained article according to the criteria described in $\operatorname{Cox}^{12,14)}$ and using the rating categories provided in Table 2. Effect sizes were also computed for each study to provide precise statistical evidence for a clinical recommendation.

\section{Quality Assessment}

The quality of the identified studies was evaluated by applying the following criteria: 1) randomization procedure; 2) blinding; and 3) number of participants. A summary of the quality of the identified studies is offered in Table 3. Of the four studies, one used a randomized crossover design, while the oth- er three used a non-randomized intervention design. This led to a level of evidence rating of 2 and 3, respectively, based on the categories listed in Table 2. All four studies were single blinded. Three of the studies had more than ten participants. Regarding potential weakness, one study had only nine participants; participants' aided localization performance in two of the studies was evaluated without acclimatization (Table 3).

\section{Outcome Measures}

The outcome measures used in the four studies included objective lab-based localization performance measurements and subjective self-report measurements through which real-world performance was assessed. Results from the identified studies were reviewed based on these two outcome measure categories. In the studies, advantages of using PPT were evaluated by comparing the outcomes using PPT to the outcomes using omni-directional and full directional microphone settings. In addition, the advantages of using PPT were further demonstrated by using an effect size analysis ${ }^{15)}$ for inter-study comparisons. For readers who may not be familiar with the concept of effect size, it is a way to display standardized magnitude of a difference by comparing the findings from different studies. There are many ways to compute an effect size. Interested readers can refer to Lipsey and Wilson ${ }^{16)}$ for more details. In the present review, a standardized mean difference effect size was used. This value also is referred to Cohen's $d$ and is computed by dividing the difference between two group means by the pooled standard deviation of the two groups. According to Cohen, ${ }^{15)}$ Cohen's $d$ values that are no more than 0.2 are considered to constitute a small effect; values that are around 0.5 are considered to be a moderate effect, while values that calculate as being no less than 0.8 are considered a large effect.

\section{Lab-based localization performance}

In the selected studies that were analyzed for this review, objective horizontal localization performance was examined in either an anechoic chamber or a sound-treated booth. A loudspeaker array was used for an evaluation of front/back localization performance. Test stimuli were presented from the front and also from the back of the test participant with or without masking noises. Test participants reported the direction after the presentation of each test stimulus. Root-mean-square (RMS) error is a performance measure reported in these studies. Computation of an RMS error is thoroughly described in Van den Bogaert, et al. ${ }^{5)}$ In general, the results from the selected studies showed that using PPT yielded less front/back confusion compared to using omni-directional or full directional settings (Table 1). These lab-based data supported the efficacy of PPT and 


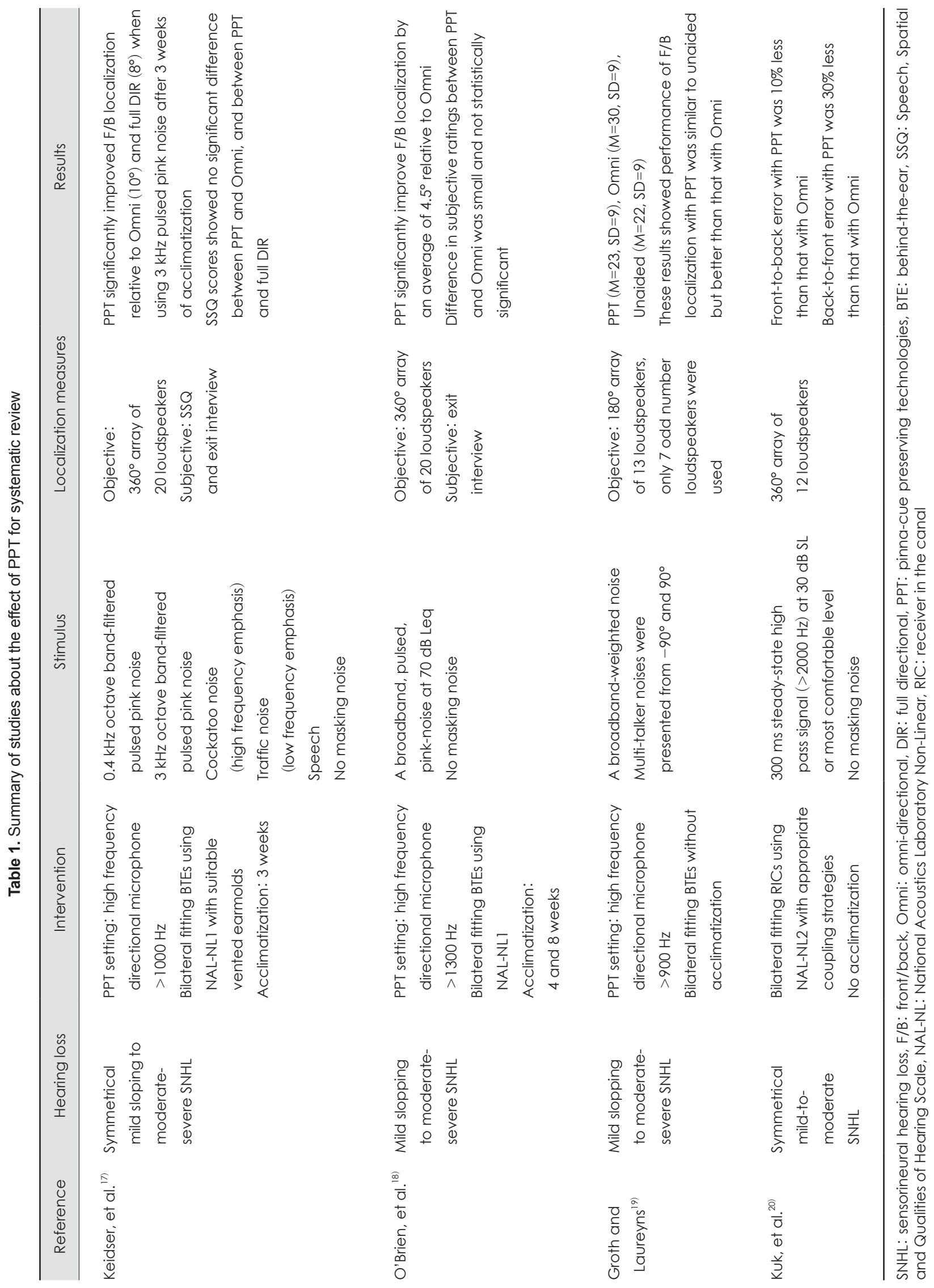


provided an internal validity of the findings. The details for each of the four studies are elaborated on below.

Keidser, et al. ${ }^{17)}$ evaluated the effectiveness of PPT used in Siemens Acuris S hearing aids. Lab-based localization performance was assessed using five different stimuli with different frequency emphases (Table 1) in quiet. They found statistically significant benefits in using the PPT setting relative to both the omni-directional and full directional settings when using stimuli with high frequency emphasis after a 3-week acclimatization period. Across the five-test stimuli, mean RMS front/ back errors with PPT were $0.7-13.2^{\circ}$ smaller than those with the omni-directional setting and $2.9-7.1^{\circ}$ smaller than those with the full directional setting. The advantages of using PPT showed large effects when test stimuli were with sufficient high

Table 2. Levels of evidence*

\begin{tabular}{cl}
\hline Level & Type of evidence \\
\hline 1 & $\begin{array}{c}\text { Systematic reviews and meta-analyses of } \\
\text { randomized controlled trials }\end{array}$ \\
2 & Randomized controlled trials \\
3 & Non-randomized intervention studies \\
4 & Descriptive studies (cross-sectional surveys, \\
& cohort studies, case-control designs) \\
6 & Case studies \\
*adapted based on Table 1 in Cox
\end{tabular}

frequency components, i.e., $3000 \mathrm{~Hz}$ pink noise, cockatoos, and speech (Table 4). Compared to the full directional setting, the PPT setting showed a moderate to large effect for all test stimuli (Table 4).

The findings of Keidser, et al. ${ }^{17)}$ were endorsed by another study conducted by O'Brien, et al. ${ }^{18)}$ In this later study, the effect of PPT used in Phonak Naida V SP hearing aids was assessed. Front/back localization tests were conducted in a quiet environment after one, four, and eight weeks of acclimatization. Taking acclimatization into consideration, this current review used the results based on the data collected after four and eight weeks. These results showed that front/back RMS error with PPT was on average $4.5^{\circ}$ less than that with the omni-directional setting. Moreover, the improvement in front/back localization was greater after 4 weeks than after 8 weeks of acclimatization. Similar to Keidser, et al., ${ }^{17)}$ a large effect size was obtained when it was measured after 4 weeks of acclimatization. Such an effect was reduced to a medium effect size, however, after 8 weeks of acclimatization.

Groth and Laureyns ${ }^{19)}$ evaluated the effect of PPT used in ReSound Live 71D hearing aids. Front/back localization performance with PPT was compared to the performance under omni-directional and unaided conditions. In that study, listeners' front/back localization performance was evaluated in the presence of a masking noise (Table 1). Unfortunately, the pre-

Table 3. Summary of quality of the identified studies

\begin{tabular}{|c|c|c|c|c|c|}
\hline Study & Randomization procedure & Blinding & $\begin{array}{l}\text { Number of } \\
\text { participant }\end{array}$ & $\begin{array}{l}\text { Weakness } \\
\text { of the study }\end{array}$ & $\begin{array}{c}\text { Level of evidence } \\
\text { according to Cox } \\
\text { (2005) }\end{array}$ \\
\hline Keidser, et al. ${ }^{17)}$ & Randomized crossover & Single & 21 & & 2 \\
\hline O'Brien, et al. ${ }^{18)}$ & Non-randomized intervention & Single & 23 & & 3 \\
\hline $\begin{array}{l}\text { Groth and } \\
\text { Laureyns }^{19)}\end{array}$ & Non-randomized intervention & Single & 14 & No acclimatization & 3 \\
\hline Kuk, et al. ${ }^{20)}$ & Non-randomized intervention & Single & 9 & $\begin{array}{l}\text { Small number of participants } \\
\text { No acclimatization }\end{array}$ & 3 \\
\hline
\end{tabular}

Table 4. Effect of PPT using Cohen's d

\begin{tabular}{|c|c|c|c|c|}
\hline \multirow{2}{*}{ Study } & \multicolumn{2}{|c|}{ Lab-based tests } & \multicolumn{2}{|c|}{ Self-report outcome } \\
\hline & PPT vs. Omni & PPT vs. DIR & PPT vs. Omni & PPT vs. DIR \\
\hline Keidser, et al. ${ }^{17)}$ & $\begin{array}{l}3 \mathrm{kHz}: 1.51 \\
0.4 \mathrm{kHz}: 0.99 \\
\text { Cockatoos: } 2.13 \\
\text { Traffic: } 0.14 \\
\text { Speech: } 1.43\end{array}$ & $\begin{array}{l}3 \mathrm{kHz}: 0.97 \\
0.4 \mathrm{kHz}: 0.50 \\
\text { Cockatoos: } 0.98 \\
\text { Traffic: } 0.75 \\
\text { Speech: } 0.68\end{array}$ & $\begin{array}{l}\text { SSQ spatial: }-0.38^{*} \\
\text { Performance rating: } 0\end{array}$ & $\begin{array}{l}\text { SSQ spatial: }-0.37^{*} \\
\text { Performance rating: } 0.30\end{array}$ \\
\hline O'Brien, et al. ${ }^{18)}$ & $\begin{array}{l}\text { After } 4 \text { weeks: } 1.00 \\
\text { After } 8 \text { weeks: } 0.40\end{array}$ & NA & Performance rating: 0.15 & NA \\
\hline Groth and Laureyns ${ }^{19)}$ & 0.78 & NA & NA & NA \\
\hline Kuk, et al. ${ }^{20)}$ & $\begin{array}{l}\text { When sounds from front: } 0.46 \\
\text { When sounds from back: } 1.08\end{array}$ & NA & NA & NA \\
\hline
\end{tabular}

*a negative Cohen's d indicates that performance with PPT was poorer than that with the microphone setting for comparison. PPT: pinna-cue preserving technologies, Omni: omni-directional, DIR: full directional 
sentation level of the masking noise was not reported by the authors. The effect of the masking noise also was not discussed in that study. It is impossible to estimate the influence of having masking noise in a localization test. Nevertheless, results showed that the percentage of front/back error was $22 \%$ with PPT and was $30 \%$ with the omni-directional setting. Performance with PPT was similar to unaided, but significantly better than performance with the omni-directional setting. Compared to the omni-directional setting, an effect size of 0.78 was observed.

A recent study by Kuk, et al., ${ }^{20)}$ evaluated PPT used in Widex Clear 440 Passion hearing aids. The PPT setting was only compared with an omni-directional setting in quiet. Front/back localization in quiet was divided into two parts, namely, frontto-back error and back-to-front error. The former was the percentage of responses that reported sounds perceived from the back when the sounds were presented from the front. The latter was the opposite. A high frequency emphasis signal was used as the test stimulus. Data were collected right after hearing fitting. The results showed that the PPT setting was significantly superior to the omni-directional setting. In terms of frontto-back error, a moderate effect of 0.46 was obtained. In terms of back-to-front error, a large effect of 1.08 was obtained. These findings indicated that PPT had advantages over omni-directional settings in front/back discrimination, especially when sounds emitted from the back.

\section{The self-reported measure}

After reviewing the evidence obtained in the lab-based tests, we examined the evidence obtained in the real world. Such measure was often referred to as effectiveness and measured using questionnaires or interviews. Self-report measures were only employed in two of the four studies: Keidser, et al. ${ }^{17)}$ and O'Brien, et al. ${ }^{18)}$ According to Table 1, the findings from the two studies showed that the benefit of using PPT in front/back localization was not observed in self-report measures when compared to omni-directional and full directional settings. The Speech, Spatial and Qualities of Hearing Scale (SSQ) ${ }^{21)}$ and an overall performance rating were used in Keidser, et al. ${ }^{17)}$ while an overall performance rating was used in O'Brien, et al. ${ }^{18)}$ The SSQ is a standard questionnaire that is designed to measure self-reported auditory disability in terms of speech hearing, spatial hearing, segregation of sounds, ease of listening, and sound quality. Because the focus of this present review was localization performance, only the subscale score for spatial hearing was used. In each of these studies, the mean rating score for the PPT was similar to the mean rating scores for omni-directional and full directional settings. None of the comparisons between PPT and other microphone settings were statistically significant. Based on the reported mean and standard deviations, we were able to compute Cohen's d values to evaluate the effect of PPT relative to omni-directional and full directional settings, respectively. These results are shown in Table 4. A small to no effect was observed. It is worth noting that, in Keidser, et al., ${ }^{17)}$ mean SSQ spatial rating scores for the PPT were lower than those for both the omni-directional and full directional settings.

\section{Analysis}

The reviewed studies measured PPT features equipped in behind-the-ear style hearing aids from different major manufacturers. Thus, the conclusions drawn from this review had good generalizability. The results showed sound evidence of using PPT to improve front/back confusion for adult listeners relative to using omni-directional and full directional microphone settings according to lab-based horizontal localization tests. The computed effect size values showed a moderate to large effect (Table 4). However, the self-report data did not show the same advantage of using PPT. The mixed findings of this review were in agreement with Keidser. ${ }^{13)}$ Her brief review concluded that the PPT was less effective in the real world than in the laboratory. The reasons of these contradicting findings were unclear. Keidser, et al. ${ }^{17)}$ suspected that it was due to insufficient acclimatization of sound localization to actual amplification in day-to-day listening. A longer acclimatization period may allow hearing aid users to establish new localization cues and improve their real-world localization performance. Another suspicion was that the lab-based measured did not reflect everyday listening environments, for instance, lack of visual cues and head movement. ${ }^{6)}$ Furthermore, existing standard questionnaires or overall performance ratings might not be sensitive enough to reflect specific changes in front/back localization performance, especially in complex acoustic environments, like real world listening situations. These suspicions suggest interpretation of the obtaining results with caution.

In terms of clinical applications, the overall findings of the present review suggested that PPT is not a critical factor to consider when prescribing hearing aids for an adult patient in the real world. However, this finding did not mean to leave PPT out of consideration when prescribing hearing aids. It is worth noting that in the two studies that included self-report measures, individual variations on the reported benefit of using PPT were observed. This indicated that some of the participants actually did notice a benefit from using PPT in their everyday lives. Despite the fact that the acclimatization effect, as suggested by Keidser, et al., ${ }^{17)}$ might indeed come into play, such observations also suggested that at least some hearing aid us- 
ers could potentially benefit from using PPT in terms of front/ back localization. Thus, the question becomes how to determine whether a given patient is a candidate for PPT.

The determination of PPT candidacy is complex, and the criteria are unknown. According to the results of this review, it is assumed that hearing aid users whose hearing loss is similar to the test participants in the reviewed studies and their major everyday communication situations are similar to a lab environment are potentially able to receive benefits from using PPT. Therefore, assessment of PPT candidacy requires a full consideration of a variety of variables based on the reviewed studies. The first variable is hearing loss, which is the basic information about a patient with hearing problems. It was noticed that the reviewed evidence was collected from adult listeners with mild sloping to moderate or to moderate-severe sensorineural hearing loss. That is to say, hearing loss of the research participants was mainly at high frequencies. Previous research has shown that listeners with sloping high frequency hearing loss find localization to be more important than their counterparts with a flat hearing loss. ${ }^{1}$ This finding suggests that, when compared to listeners with a flat hearing loss, listeners with a sloping high-frequency hearing loss are more likely to have a need to improve their aided localization performance. Thus, if a patient has mild sloping to moderate or moderate-severe sensorineural hearing loss, he/she meets the first criterion for PPT candidacy.

Based on the reviewed evidence, we concluded that PPT was effective when the hearing aids were working in a well-controlled lab environment and the target sounds contained sufficient high frequency components. These findings suggest that two additional criteria need to be met in order for clients to benefit from PPT: 1) a relatively quiet environment (given that the lab-based localization test in one of the four studies was conducted with masking noise) without much reverberation and 2) sounds containing sufficient high frequency components. That is, if a patient reports hearing undertaken in a relatively quiet situation often in his/her daily life and his/her major communication environments are not reverberant, then that patient is likely to benefit from using PPT. In addition, if the sounds that the patient needs to locate have substantial high frequency components, then this patient also is likely to benefit from using PPT. Information regarding the communication environment and the specific sound characteristics for localization can be obtained from each patient via a pre-fitting interview or a consultation. A thorough interview or consultation can thus provide valuable information about the patient, including life style, communication environments, communication partners, and communication situations that are important to the patient. The information needed for assessment of PPT candidacy was then used to determine whether a patient is a candidate.

The abovementioned criteria for PPT candidacy were supported by a study conducted by Jensen, et al., ${ }^{6}$ in which benefits of using pinna-cue preserving hearing aids were assessed. Because the pinna cue preserving setting that they evaluated was realized by physically relocating the BTE hearing aid microphone to the entrance of the ear canal instead of digitally restoring the directivity of a pinna, that study was excluded from this review. However, Jensen and colleagues did address some of the issues regarding localization cues provided by a pinna. In that study, lab-based localization testing and a self-report measurement were performed. They concluded that preserving pinna cues could provide benefit under certain conditions for hearing aid users with mild to moderate hearing loss.

When PPT candidacy is determined, appropriate hearing aids are then selected on the basis of factors that are normally considered in a clinical hearing aid prescription protocol along with PPT. It is important to note that the benefit of using PPT could not be guaranteed for each PPT candidate. Hearing aid orientation and consultation are crucial to informing the patient on how to make use of PPT in real world situations and helping the patient establish realistic expectations for using amplification. It is a mistake to select hearing aids for a PPT candidate based solely on PPT availability. PPT should be considered as an additional feature that can potentially benefit certain hearing aid users in some listening environments. Audiologists should make good use of the collected information from the pre-fitting interview/consultation to better understand each patient's goals for amplification and then select the appropriate hearing aids or hearing aid features that best suit the patient's daily needs. In addition, aural rehabilitation should not be ignored to maximize hearing aid success. ${ }^{22,23)}$

\section{Conclusions and Recommendations}

PPT is designed to mimic the frequency-specific directivity of the human outer ear by using digital signal processing to improve front/back discrimination. The findings reported here after a systematic review of four studies indicated that this feature could yield a substantial reduction in front/back confusion for adult hearing aid users when those users were evaluated in laboratories. However, the advantages of using PPT could not be observed in the real world. Thus, it appears that PPT is advantageous in a lab environment, but it has limited real-world application. However, the observed individual difference in real-world performance did suggest that some hearing aid users could potentially benefit from using PPT to improve their front/back sound discrimination performance. It is important to conduct a pre-fitting interview/consultation to gather infor- 
mation regarding a given patient for determination of PPT candidacy. The collected information also will assist audiologists in making the best evidence-based and cost-effectiveness decisions when prescribing hearing aids to their patients.

\section{REFERENCES}

1) Meister H, Lausberg I, Kiessling J, Walger M, von Wedel H. Determining the importance of fundamental hearing aid attributes. Otol Neurotol 2002;23:457-62.

2) Blauert J. Spatial hearing: The psychophysics of human sound localization. Cambridge, MA: MIT Press;1997.

3) Van den Bogaert T, Klasen TJ, Moonen M, Van Deun L, Wouters J. Horizontal localization with bilateral hearing aids: without is better than with. J Acoust Soc Am 2006;119:515-26.

4) Köbler S, Rosenhall U. Horizontal localization and speech intelligibility with bilateral and unilateral hearing aid amplification. Int J Audiol 2002;41:395-400.

5) Van den Bogaert T, Carette E, Wouters J. Sound source localization using hearing aids with microphones placed behind-the-ear, in-thecanal, and in-the-pinna. Int J Audiol 2011;50:164-76.

6) Jensen NS, Neher T, Laugesen S, Johannesson RB, Kragelund L. Laboratory and field study of the potential benefits of pinna cue-preserving hearing aids. Trends Hear 2013;17:171-88.

7) Van den Bogaert T, Doclo S, Wouters J, Moonen M. The effect of multimicrophone noise reduction systems on sound source localization by users of binaural hearing aids. J Acoust Soc Am 2008;124:484-97.

8) Sivonen VP. Binaural directivity patterns for normal and aided human hearing. Ear Hear 2011;32:674-7.

9) Upfold LJ, May AE, Battaglia JA. Hearing aid manipulation skills in an elderly population: a comparison of ITE, BTE, and ITC aids. Br J Audiol 1990;24:311-8.

10) Kirkwood DH. Resilient hearing aid industry records rising sales de- spite a troubled economy. Hear J 2009;62:11-6.

11) Strom K. Sales increase by $3 \%$ in 2011. Hear Rev 2012;19:6.

12) Cox RM. Evidence-based practice in provision of amplification. J Am Acad Audiol 2005;16:419-38.

13) Keidser G. Evidence-based practice and emerging new technologies. In: Wong L, Hickson LM, editors. Evidence-based practice in audiology: Evaluating Interventions for Children and Adults with Hearing Impairment. San Diego, CA: Plural Publishing;2012. p.119-37.

14) Cox RM. Waiting for evidence-based practice for your hearing aid fittings? It's here! Hear J 2004;57:10-7.

15) Cohen J. Statistical Power Analysis for the Behavioral Sciences. Hillsdale, NJ: L. Erlbaum Associates;1988.

16) Lipsey MW, Wilson DB. Practical meta-analysis (applied social research methods). Thousand Oaks, CA: Sage Publications, Inc.;2001.

17) Keidser G, O'Brien A, Hain JU, McLelland M, Yeend I. The effect of frequency-dependent microphone directionality on horizontal localization performance in hearing-aid users. Int J Audiol 2009;48:789803.

18) O'Brien A, Yeend I, Hartley L, Keidser G, Nyffeler M. Evaluation of frequency compression and high-frequency directionality. Hear J 2010;63:32-7.

19) Groth J, Laureyns M. Preserving localization in hearing instrument fittings. Hear J 2011;64:34-8.

20) Kuk F, Korhonen P, Lau C, Keenan D, Norgaard M. Evaluation of a pinna compensation algorithm for sound localization and speech perception in noise. Am J Audiol 2013;22:84-93.

21) Gatehouse S, Noble W. The Speech, Spatial and Qualities of Hearing Scale (SSQ). Int J Audiol 2004;43:85-99.

22) Hawkins DB. Effectiveness of counseling-based adult group aural rehabilitation programs: a systematic review of the evidence. J Am Acad Audiol 2005;16:485-93.

23) Sweetow R, Palmer CV. Efficacy of individual auditory training in adults: a systematic review of the evidence. J Am Acad Audiol 2005; 16:494-504. 